\title{
Lateral pedicle graft: A treatment modality for root coverage of localized gingival recession: A case report
}

\author{
Parul $^{1}$, Pujit Chhabra ${ }^{2, *}$, Amisha $^{3}$, Monika $^{4}$ \\ ${ }^{\mathbf{1}}$ Periodontist, ${ }^{\mathbf{P}} \mathrm{PG}$ Student Orthodontist, ${ }^{\mathbf{3}} \mathrm{PG}$ Student Oral and Pathology, ${ }^{\mathbf{4}} \mathrm{PG}$ Student Periodontics, ${ }^{\mathbf{1}, \mathbf{4}}$ Dept. of Periodontics, \\ ${ }^{2}$ Dept. of Orthodontics, ${ }^{3}$ Dept. of Oral and Pathology, ${ }^{\mathbf{1 , 2}, 4} \mathrm{Jan}$ Nayak Ch. Devi Lal Dental College Sirs, Haryana, ${ }^{3}$ Surender Dental \\ College Rajasthan, India
}

*Corresponding Author:

Email: drpujitchhabra@gmail.com

\begin{abstract}
Gingival recession has been defined as the apical shift of gingival margin from its physiologic level (1 to $2 \mathrm{~mm}$ coronal to cementoenamel junction) causing pathologic exposure of root surfaces. Mucogingival esthetic surgery is all about treatment of mucogingival esthetic alterations. Mucogingival problems may be caused by gingival recession often in association with noncarious cervical lesion or by root caries or by altered passive eruption. Patient with gingival recession frequently report to dental clinics which could lead to pain or hypersensitivity, esthetic problem, retention of plaque hence inflamed gingiva, root caries, abrasion and fear of tooth loss. Gingival recession can be treated by surgical or non-surgical means. Nonsurgical means include restorations, crowns, veneers and gingival masks whereas surgical approaches includes various methods of increasing the width of keratinized tissue such as frenectomy in case of high frenal attachment \& root coverage procedures.

The lateral displaced pedicle flap was first described by Grupe and Warren in 1956. It was used to cover the isolated, denuded root surfaces that have adequate amount of donor tissue adjacent to the recipient site. The vestibular depth must be adequate to laterally slide the pedicle. A case of gingival recession is presented that was managed using LPG technique. This technique was selected because of certain advantages - single surgical area, preservation of blood supply of flap, the postoperative color being in harmony with surrounding tissue.
\end{abstract}

Keywords: Lateral pedicle, Graft, Gingival recession.

\section{Introduction}

Gingival recession is a mucogingival defect of multifactorial origin. In 1956 Grupe and Warren developed an original and unique procedure called the sliding flap operation for covering an isolated exposed root. LPG is a technique where graft is elevated from donor site which remains attached at its base for nourishment and is transferred to adjacent site in isolated denuded root. ${ }^{1,2}$ To prevent donor site recession, Grupe (1966) modified this to a sub marginal incision on the donor site. Staff leno (1964) solved this problem by using a partial-thickness flap to protect the donor site from recession. Corn (1964) further modified this by adding a cutback incision to release tension. Dahlberg (1969) used engineering principles with the rotated pedicle flap, which did not require acutback incision. Goldman and Smukler (1978) added the periosteally stimulated flap and a partial-full rotated flap in 1983 which allowed a full-thickness flap to cover the denuded root surface and a partial-thickness flap to cover the exposed bone. ${ }^{3}$

\section{Case Report}

A 23 years old female patient came to Dental clinic with chief complaint of unaesthetic appearance, sensitivity in lower front region, receding gums from last past 7-8 months. Nothing significant reported in past dental history and medical history. On intraoral examination periodontal pockets were absent. There was Miller's Class II recession on 31 (Fig. 1,2) and no Trauma from occlusion was present.
Phase I therapy was completed and patient was recalled after 4 weeks for evaluation. After patient's consent it was decided to treat the case surgically by lateral (horizontally) displaced flap.

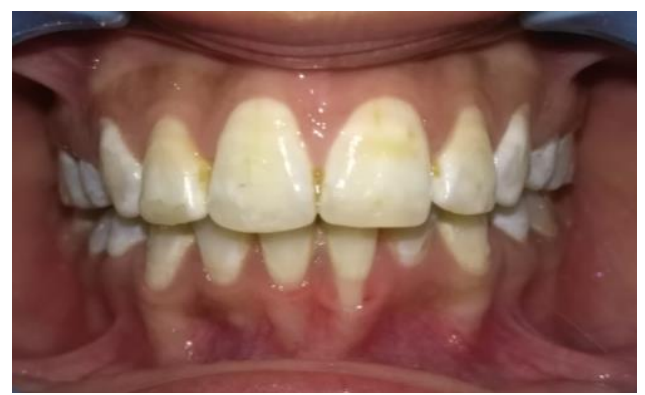

Fig. 1: Pre - operative photograph

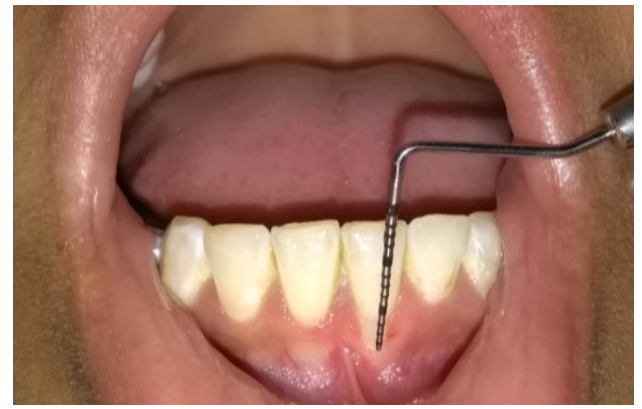

Fig. 2: Pre - operative photograph with periodontal probe 


\section{Surgical Technique}

Preparation of Recipient Site: After proper isolation of surgical site, Local anesthesia 1:80,0000 was administered. A 15 no. scalpel blade is used to make a $\mathrm{V}$-shaped incision along the soft tissue margin of the recipient site, removing the adjacent epithelium and connective tissue, with reverse bevel incision on the gingival margin adjacent to the donor site (i.e. margin close to 31) and an external bevel on the opposite margin (adjacent to 41) to remove the epithelium around the denuded root surface. The exposed connective tissue adjacent to distal margin of 31 was to be the recipient site for laterally displaced flap taken from site of 32 .

Preparation of Flap: Periodontium of donor site had adequate width of attached gingiva with minimal loss of alveolar bone, no dehiscence and fenestration. With a 15 no. Scalpel blade, partial thickness flap was raised and a vertical incision was given from gingival margin to outline a flap adjacent to the recipient site Incision, and extended to the level of the base of the recipient site.

The flap was wider than the recipient site to cover the denuded root and provide a broad margin for attachment to the connective tissue. A cut back incision was made into alveolar mucosa at the distal corner of flap pointing towards recipient site. (Fig. 3).

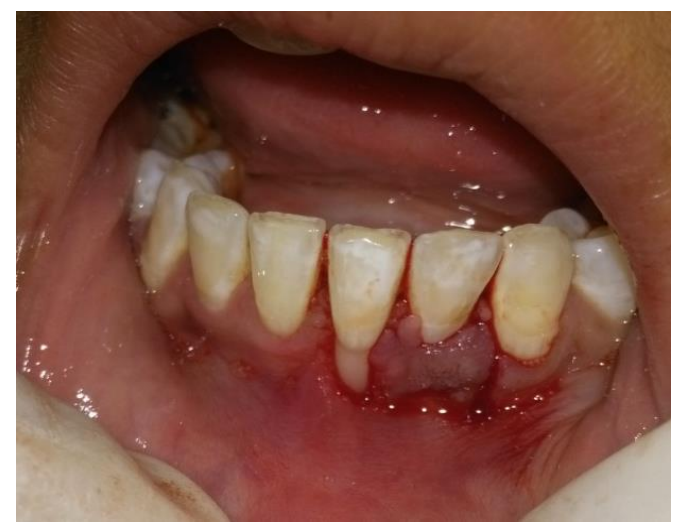

Fig. 3: Preparation of recipient site

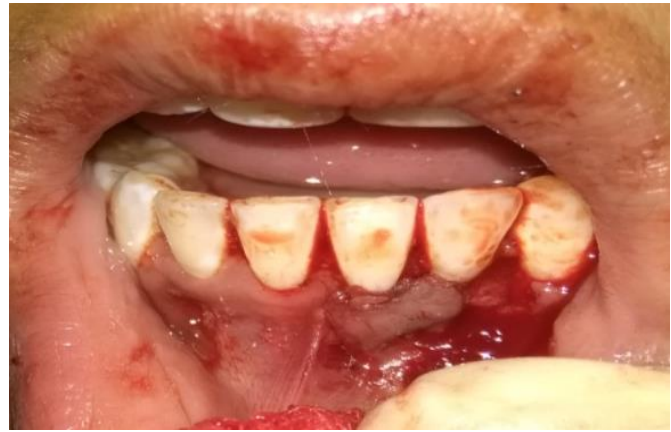

Fig. 4: Transfer of flap

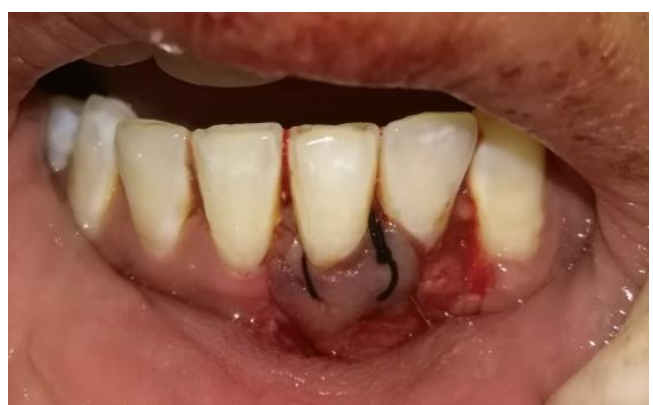

Fig. 5: Flap in position and sutured

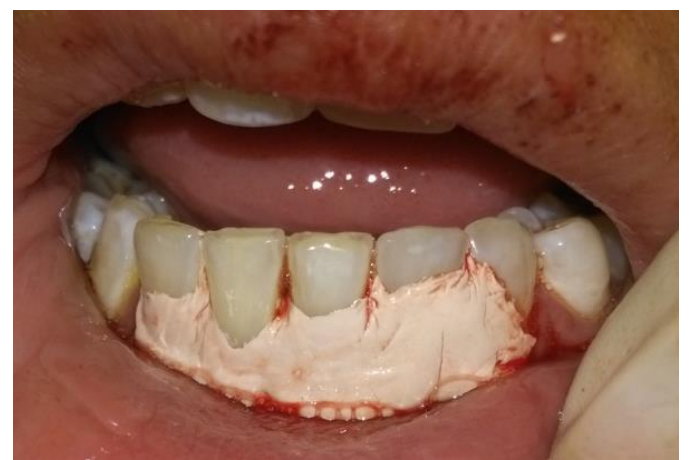

Fig. 6: Periodontal dressing

Transfer of Flap: Flap was shifted laterally to the adjacent denuded root (Fig. 4) making it adapted appropriately and without tension. Flap was fixed with interrupted sutures to prevent slipping it apically (Fig. 5).

Placement of Periodontal Pack: The surgical site was covered periodontal pack (Coe-Pak) (Fig. 6). The patient was recalled after 1 week for pack and suture removal.

Postoperative Instructions: To prevent infective complications, antibiotics were prescribed to patients. Amoxycillin $500 \mathrm{mg}$ thrice a day for 5 days and Analgesics (Ibuprofen) was prescribed thrice a day for 3 days and $0.2 \%$ Chlorhexidinedi gluconate mouth wash was prescribed twice daily for four weeks and advised to avoid vigorous brushing on the surgical site. The patient was recalled for follow-up after 1 week. The pack and sutures were removed and site was irrigated with normal saline and Betadine. Healing was satisfactory. Recall was scheduled for 3 months. The recession coverage result showed morphologic and chromatic resemblance to adjacent gingiva. Thus patient satisfaction was perceived (Fig. 7). 


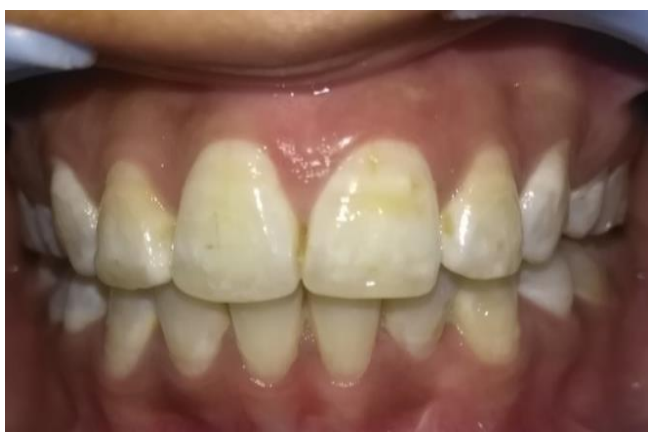

Fig. 7: Post-operative photograph after 3 months

\section{Discussion}

Over years, several techniques are used to obtain root coverage. but advantage of this technique over other is morphologic and chromatic resemblance its simplicity, presence of single surgical site and good vascularity of pedicle. ${ }^{4}$ Disadvantages include probable recession, dehiscence or fenestration at donor site and its limitation to only one or two teeth. Indications for lateral pedicle flap are sufficient length, width and thickness of keratinized tissue, recession limited to 1-2 teeth, adequate depth of vestibule and narrow recession. Contraindications are insufficient length width and thickness of keratinized tissue presence of fenestration or dehiscence at donor site, exceptionally protrusive teeth deep periodontal pockets loss of interdental bone and inadequate depth of vestibule. ${ }^{5}$ This technique was evaluated by many investigators (McFall $1967 .{ }^{6}$ Smukler $1976 .{ }^{7}$ ) and the success of this root coverage procedure was found to be in the range of $69 \%$ to $72 \%$. Causes of failure to cover the denuded root surface could be tension at base of distal incision, too narrow pedicle full thickness flap to cover might lead to exposure of bone which leads to bone loss and poor stabilization \& mobility of the graft. ${ }^{5}$

Similar study was done by Verma PK results showed acceptable root coverage of miller's Class II recession with LPG in the lower anterior teeth. ${ }^{8}$ Similarly Chopra DK et al. did LPG in mandibular anterior area for the treatment of Class-III gingival recession and esthetic root coverage was obtained with no alteration in the position of gingiva at the donor site. ${ }^{9}$ And Gupta $\mathrm{S}$ et al. performed LPG in patient with Miller's Class III recession on 41 with labial frenal pull and obtained acceptable root coverage.$^{10}$ Kritika et al. in 2018 performed similar case to cover root recession using LPG with sub marginal incision and obtained predicable results. ${ }^{11}$

\section{Source of Support and Sponsorship: Nil}

\section{Conflicts of Interest: None.}

\section{References}

1. Lindhe J, Lang NP, Karring T. Clinical periodontology and implant dentistry: Wiley-Blackwell. 2009.

2. Newmann MG, Takei HH, Klokkevold PR, Carranza FA. Carranza's Clinical Periodontology. $10^{\text {th }} \mathrm{Ed}$. Philadelphia: W.B. Saunders Co. 2002;639-42.

3. Naoshi Sato. Periodontal surgery: A clinical atlas. Quintessence. 2000.

4. Kumar A, Jan SM, Behl R, Nazir R, Khairat RU, Ganaie TA. Laterally positioned flap with periosteal graft for the treatment of gingival recession, novel technique - A pilot study. IAIM. 2016;3(6):192-98.

5. Rajkarnikar J. Efficacy of Lateral Pedicle Graft in the Treatment of Isolated Gingival Recession Defects. Int $J$ of Pharmaceutical Science Invention. 2014;3(1):46-50.

6. McFall W Jr. The laterally repositioned flap. Period. 1967;5:89-95.

7. Smukler H. Laterally positioned mucoperiosteal pedicle grafts in the treatment of denuded roots. Period. 1976;47:590-95.

8. Verma PK. Esthetic root coverage of Miller's Class II recession with laterally positioned flap. 2012;2:75-8.

9. Chopra DK, Kaushik M, Kochar D, Malik S. Laterally Positioned Flap - A Predictable and Effective Periodontal Procedure for the Treatment of Adjacent Class-III Gingival Recession Defect - Case Report. JIDA. 2011;5(6):725-27.

10. S Gupta, S Pardhan. Lateral Pedicle Graft: A treatment modality for rootcoverage in the management of localized gingival recessions. J of Nepal Dent Assoc (JNDA). 2015;15(1).

11. Kritika, Pardeep S, Attresh G. gingival recession coverage by lateral pedicle procedure: case report. IJRDO. 2018;4(9).

How to cite this article: Parul, Chhabra P, Amisha, Monika. Lateral pedicle graft: A treatment modality for root coverage of localized gingival recession: A case report. Int J Periodontol Implantol. 2018;3(4):134-136. 